[7] H. M. Barakat, A. R. Omar, and O. M. Khaled, A new flexible extreme value model for modeling the extreme value data, with an application to environmental data, SATAT. PROBABIL. IETT. (2017), doi.org/10.1016/j.spl.2017.07.002

[8] G. Box, D. Cox, An analysis of transformations. J. R. Stat Soc. B. (1964) 211264.

[9] NR. Mohan, S. Ravi Max domains of attraction of univariate and multivariate p-max stable laws. Theor. Probability Appl. 37 (1992) 632-643.

[10] E. Pantcheva, Limit theorems for extreme order statistics under nonlinear normalization. Lect. Not. Math. 1155 (1984) 284-309.

[11] U. R. Subramanya, On max domains of attraction of univariate p-max stable laws. Stat. Probability. Lett., 19 (1994) 271-279.

[12] G. Christoph, and M. Falk, A note on domains ofattraction of p-max stable laws. Stat. Probability. Lett. 28 (1996) 279-284.

[13] G. Fuller, P. Johnson, A. Cue, Air quality in London 2001:the ninth report of the London Air Quality Network. London, Environmental Research Group, Kings College London, 2003.

[14] C. David Carslaw, D. Sean Beevers and Gary Fuller .An empirical approach for the prediction of annual mean nitrogen dioxide concentrations in London,Atom. Environ. 35 (2001) 1505-1515

[15] R. Maheswaran, T. Pearson, SD. Beevers ,MJ. Campbell, CD. Wolfe Air Pollution and Subtypes, Severity and Vulnerability to IschemicStrokeA Population Based Case-Crossover Study. Plos One 11(6) (2016) e0158556. doi: 10.1371/journal.pone.0158556.: 


\title{
Thermosolutal Marangoni Convection Effect on the MHD Flow over an Unsteady Stretching Sheet
}

By

\author{
Mohamed A. Hassan ${ }^{1}$
}

Department of Mathematics, Faculty of Education, Ain Shams University, Roxy, Cairo, Egypt

Received 17/2/2017

Revised 28/5/2017

Accepted 14/91/2017

\begin{abstract}
The unsteady magnetohydrodynamic (MHD) thermosolutal Marangoni convection flow with heat and mass transfer over an unsteady stretching sheet is examined. The surface tension is assumed a linear function of the temperature and concentration. The quasilinearization method (QLM) is applied with employing the method of complementary function and the fourth order Runge-Kutta method to the yielding ordinary nonlinear equations. The analytical solution for the steady flow, with some special values of the problem parameters, is compared with the present numerical results. The results show that the decreasing of the surface tension enhances the flow movement due to decreasing the free surface rigidity. Also, the enhancement of the temperature provokes the free surface and reduces the surface tension. This result is in agreement with the previous experimental published results. Also, the flow velocity increases with reducing the surface tension. Meanwhile, the concentration surface tension coefficient reduces the concentration profiles. Finally, the numerical solution is compared with the previous published results for a special choice of the parameters. The graphical and tabulated results reveals on the high fidelity of the numerical method.
\end{abstract}

Keywords: Marangoni effect; Magnetohydrodynamic; Heat transfer; mass transfer; Unsteady flow; Stretching Sheet; Numerical quasilinearization method.

\section{Classification No.: 76.}

\section{Nomenclature}

$b \quad$ positive constant in Eq. (7). $[1 / \mathrm{sec}]$

$B_{0} \quad$ strength of the magnetic field. $\left\lfloor\mathrm{Wb} / \mathrm{m}^{2}\right\rfloor$

$C$ concentration. [-]

$c$ positive constant. $[1 / \mathrm{sec}]$

$C f_{x} \quad$ local skin friction. [-]

$c_{p} \quad$ specific heat capacity. $[\mathrm{J} /(\mathrm{kg} . K)]$

$c_{m} \quad$ concentration susceptibility. $\left[\mathrm{J}^{-1} . \mathrm{kg}\right\rfloor$

$D_{m} \quad$ mass diffusivity. $\left\lfloor\mathrm{m}^{2} / \mathrm{sec}\right\rfloor$

$f \quad$ non-dimensional stream function. $[-]$

$$
\begin{array}{lc}
T_{m} & \text { mean fluid temperature }[K] \\
u & x \text {-axis velocity component. }[\mathrm{m} / \mathrm{sec}] \\
v & y \text {-axis velocity component. }[\mathrm{m} / \mathrm{sec}] \\
x & \text { horizontal axis along the plate. }[\mathrm{m}] \\
y & \text { vertical axis normal to the plate. }[\mathrm{m}]
\end{array}
$$

\section{Greek symbols}

$\sigma \quad$ variable surface tension. $\left[\mathrm{m} / \mathrm{sec}^{2}\right]$

$\sigma_{0} \quad$ constant value of the surface tension. $\left\lfloor\mathrm{m} / \mathrm{sec}^{2}\right\rfloor$

$\gamma_{T} \quad$ temperature coefficient of the surface tension. $\left[K^{-1}\right\rfloor$

$\gamma_{C}$ concentration coefficient of the surface tension. [-]

E-mail address: mohamed_gaber@edu.asu.edu.eg 


$\begin{array}{ll}h & \text { liquid film thickness. [m] } \\ k & \text { thermal conductivity. [W/m.K] } \\ k_{T} & \text { thermal diffusion ratio. [-] } \\ M & \text { magnetic parameter. [-] } \\ m & \text { constant power in Eq.(7). [-] } \\ N u_{x} & \text { local Nusselt number. [-] } \\ N m_{x} & \text { local Sherwood number. [-] } \\ \mathrm{Pr} & \text { Prandtl number. [-] } \\ r & \text { constant power in Eq.(7). [-] } \\ \mathrm{Re}_{x} & \text { local Reynolds number. [-] } \\ S & \text { Parameter of unsteadiness. [-] } \\ t & \text { time. [sec] } \\ T & \text { temperature. [K] }\end{array}$

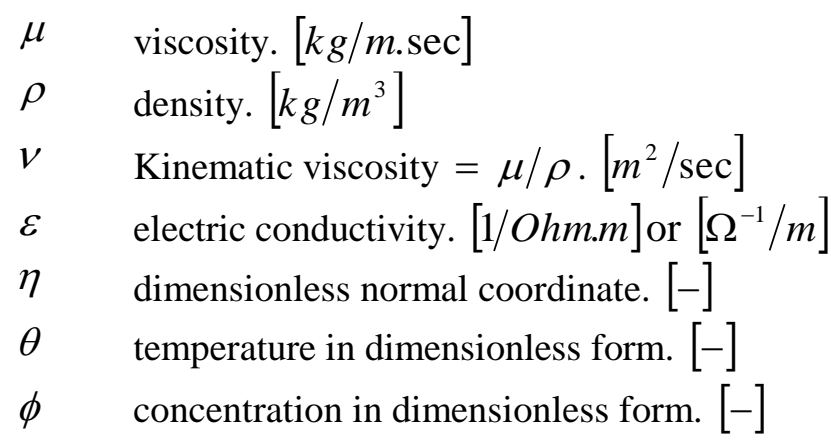

\section{Subscripts}

$\infty \quad$ value at infinity (at the free surface).

$0 \quad$ value at the slot (origin).

$s \quad$ value at the sheet.

ref reference value.

$x \quad$ local value.

\section{Introduction}

The Marangoni effect represents the surface tension variation due to the temperature gradient along the surface. The Marangoni boundary layer occurs along liquid/liquid or liquid/gas interface. The thermo-capillary flow produces a motion within the fluid according to the thermal Marangoni convection currents. The surface tension gradients depend on both temperature and/or concentration gradients. Napolitano [1,2] who is firstly studied this phenomenon. Christopher and Wang [3] analyzed the Marangoni effect for the boundary layer flow over a flat surface with temperature gradient. Meanwhile, the Marangoni convection on the power-law thin liquid film over an unsteady stretching surface with heat transfer has been explored by Chien-Hsin Chen [4]. Pop et. al. [5] studied the Marangoni boundary layer for larger values of Reynolds number. The authors assumed the temperature and the solutal concentration, at the interface, as power functions of the longitudinal coordinate.

Studying the influence of both the Marangoni convection currents and the Magnetohydrodynamic (MHD) on the flow behavior is important in many engineering applications. MHD is considered as a branch of fluid mechanics that studies the magnetic force effects. Also, MHD involves the influence of moving media (fluid flow) through a magnetic field. The study of the flow of an electrically conducting fluid has many applications in engineering problems such MHD generators, plasma physics, geothermal energy, nuclear reactors and aerodynamics. Jiao et. al. [6] studied the MHD thermosolutal Marangoni convection currents on the non-Newtonian power law fluid. Also, the authors considered the power law form for the temperature and the concentration and the linearly varying of the surface tension on both the temperature and concentration. Magyari and Chamkha [7] determined the exact analytical solutions for the velocity, temperature and concentration fields of steady thermosolutal MHD Marangoni convection. The (MHD) thermocapillary Marangoni convection non-Newtonian power-law fluid was studied by Lin et. al. [8]. The results showed that the magnetic field reduces the velocity. Also, the Marangoni number reduces the temperature.

The heat and mass transfer for the flow of a thin liquid film have many applications. For example, the heat exchangers, coating of wire and fiber, chemical processing equipment, food stuff processing and polymer processing. The difference in concentration induces the driving force of the mass transfer. This phenomenon involves molecular and convective transport of atoms and molecules. The flow of two types of viscoelastic electrically conducting fluids over a stretching sheet was studied by Cortell [9]. The results showed that the magnetic field retards velocity and enhances the 
concentration. Elgazery and Hassan [10] studied the effects of variable viscosity and thermal diffusivity on the magnetohydrodynamic flow over a horizontal stretching sheet through a porous medium. The authors showed that the film thickness approaches zero as the unsteadiness parameter approaches to its critical value. Mahmoud [11] studied the influence of thermal radiation for Newtonian liquid film with and without thermocapillary over a stretching sheet. He deduced that the film thickness increases as the thermocapillarity parameter increases. The problem of unsteady stretching surface with different physical parameters under numerous conditions was studied by many authors. Andersson et. al. [12] studied the flow of a liquid film over a stretching sheet. Also, Andersson et. al. [13] studied the influence of thermocapillarity flow and heat transfer over a horizontal stretching sheet. Magyari and Ali [14] studied the unsteady flow with heat transfer induced by a submerged stretching surface. The motion is assumed to be slowed down and the surface temperature approaches the ambient temperature continuously. Dandapat et. al. [15] analyzed the effects of variable viscosity, variable thermal conductivity and thermocapillarity on the flow and heat transfer for a laminar liquid film over a horizontal stretching sheet. A similar study was investigated to examine the effect of the internal heat generation by Elbashbeshy and Bazid [16]. The unsteady boundary layer over a stretching sheet for special distribution of the stretching velocity and the surface heat flux was investigated by Pop et. al.[17]. While, the effect of the non-uniform heat source/sink on the flow over an unsteady stretching sheet through a quiescent fluid medium was studied by Huang et. al [18]. Hayat et. al [19] considered the same problem but for the electrically conducting second grade fluid film over an unsteady stretching sheet. Mukhopadhyay [20-22] studied the problem of the unsteady flow over a porous stretching sheet with the effects of the thermal radiation and with considering the effects of slip velocity at the boundary. The same problem was presented to study the effects of chemical reaction on unsteady free convective heat and mass transfer on a stretching surface and through a porous medium by Chamkha et. al. [23]. Also, the non-conventional partial slip conditions at the stretching sheet were presented for the unsteady boundary layer stagnation-point flow with considering the heat transfer towards the stretching sheet by Layek et al. [24]. The flow and heat transfer of magneticnanofluids over a stretching surface with the aligned magnetic field was considered by Sandeep [25]. Two types on ananofluids were considered, the graphene (GP) nanoparticles embedded in water and water-ethylene glycol (EG) mixtures. Also, Sandeep et al. [26] studied the magnetohydrodynamic chemically reacting Casson and Maxwell fluids past a stretching sheet. The authors discussed the effects of the cross diffusion, non-uniform heat source/sink, thermophoresis and Brownian motion. The Cattaneo-Christov heat equation model for MHD Casson-ferrofluid with radiative heat transfer was investigated by Ali and Sandeep [27]. Jayachandra and Sandeep [28] studied the upper convected Maxwell fluid (UCM) flow across a horizontal melting surface in the presence of thermal and solutal cross-diffusion and double stratification.

This paper is devoted to study the thermosolutal Marangoni convection effect on the MHD flow over an unsteady stretching sheet. The surface tension depends linearly on both the temperature and concentration. So, the combined effects of the temperature/concentration surface tension parameters are obtained on the velocity, temperature and concentration profiles. The quasilinearization method (QLM) is used to obtain the numerical solution of the coupled nonlinear ordinary differential equations. Also, the analytical solution for the steady flow is obtained. The organization of the paper as follows: In Section 2, the problem is formulated with the appropriate similarity transformation. In Section 3 the analytical solution of the steady flow for a limiting case of the problem parameters is presented. Section 4 describes the steps of the numerical solution by using QLM. The results are discussed in Section 5 and are supported with verifications by 
tabulated comparisons with the previous publishing results and with the exact solution. In Section 6 a brief conclusion is presented.

\section{Formulation of the Problem}

The unsteady laminar thermosolutal Marangoni boundary layer flow of an electrically conducting incompressible Newtonian fluid is considered. The electric conductivity $\varepsilon$ of the fluid is considered to be constant. The stretching of the elastic sheet induces the flow and the pressure gradient is neglected. The flow film is imposed by a uniform transverse magnetic field with constant strength $B_{0}$. For small magnetic

Reynolds number, the induced magnetic field can be neglected. Also, the external electric field is neglected. The physical model and the coordinate system are depicted in Fig.1. The stretching sheet is considered to be in the positive direction of $x$ - axis with the slot at the origin and the positive $y$ - axis is taken normal to the sheet in the vertical direction.

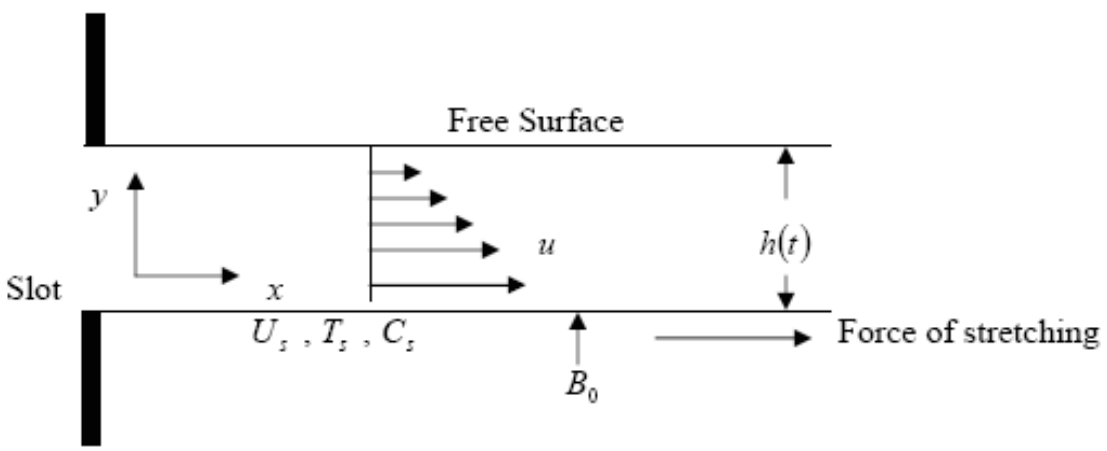

Fig.1 Schematic of the Physical model.

All physical properties of the fluid are considered constants except the surface tension $\sigma$. The surface tension is assumed a linear function of the temperature and the solute concentration. So, the surface tension depends on the temperature and concentration linearly [7], as follows:

$$
\sigma(x, y, t)=\sigma_{0}\left[1-\gamma_{T}\left(T-T_{0}\right)-\gamma_{C}\left(C-C_{0}\right)\right],
$$

where, the parameters $\gamma_{T}=-\left.\frac{1}{\sigma_{0}} \frac{\partial \sigma}{\partial T}\right|_{T}$ and $\gamma_{C}=-\left.\frac{1}{\sigma_{0}} \frac{\partial \sigma}{\partial C}\right|_{C}$ denote to the temperature and concentration coefficients of the surface tension. Also, the values $T_{0}$ and $C_{0}$ are the temperature and concentration at the slot, respectively. $\sigma_{0}$ is the constant surface tension at constant values of both the temperature and concentration. The balance between the shear stress and the tangential surface tension gradient at the free surface can be written in the form

$\left.\mu \frac{\partial u}{\partial y}\right|_{y=h}=\left.\frac{\partial \sigma}{\partial x}\right|_{y=h}=\sigma_{0}\left(\left.\gamma_{T} \frac{\partial T}{\partial x}\right|_{y=h}+\left.\gamma_{C} \frac{\partial C}{\partial x}\right|_{y=h}\right)$,

Under these assumptions, the basic equations, that govern the resulting boundarylayer flow, can be written as follows

$$
\frac{\partial u}{\partial x}+\frac{\partial v}{\partial y}=0
$$




$$
\begin{aligned}
& \frac{\partial u}{\partial t}+u \frac{\partial u}{\partial x}+v \frac{\partial u}{\partial y}=\frac{\mu}{\rho} \frac{\partial^{2} u}{\partial y^{2}}-\frac{\varepsilon B_{0}^{2}}{\rho} u, \\
& \frac{\partial T}{\partial t}+u \frac{\partial T}{\partial x}+v \frac{\partial T}{\partial y}=\frac{k}{\rho c_{p}} \frac{\partial^{2} T}{\partial y^{2}}+\frac{D_{m} k_{T}}{c_{m} c_{p}} \frac{\partial^{2} C}{\partial y^{2}}, \\
& \frac{\partial C}{\partial t}+u \frac{\partial C}{\partial x}+v \frac{\partial C}{\partial y}=D_{m} \frac{\partial^{2} C}{\partial y^{2}}+\frac{D_{m} k_{T}}{T_{m}} \frac{\partial^{2} T}{\partial y^{2}},
\end{aligned}
$$

All previous symbols are defined in the Nomenclature. The associated boundary conditions are defined as follows [11,12]:

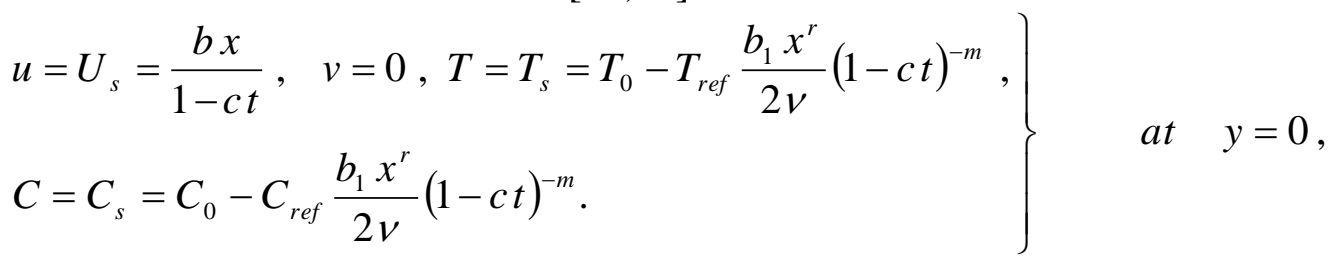

$\left.\mu \frac{\partial u}{\partial y}=-\frac{\partial \sigma}{\partial x}, \quad v=\frac{d h}{d t}, \quad \frac{\partial T}{\partial y}=0, \quad \frac{\partial C}{\partial y}=0 . \quad\right\} \quad$ at $\quad y=h$.

where, the constants $b$ and $c$ have the dimensions of time $e^{-1}$. The constant reference temperature and concentration are $T_{r e f}$ and $C_{r e f}$, respectively, which can be taken as a constant difference such that $0 \leq T_{r e f} \leq T_{0}$ and $0 \leq C_{r e f} \leq C_{0}$. The constant $b_{1}$ is a positive constant with dimensions ( Length $^{2-r}$ time $^{-1}$ ) and for the special case of the power $r=2$, the constants $b$ and $b_{1}$ are identical [29]. The powers $r$ and $m$ represent the increasing or decreasing of the temperature and the concentration with the distance from the slot. Proceeding with the analysis, the appropriate similarity dimensionless variables $f, \theta, \phi$ and $\eta$ may be written as follows [12]:

$$
\left.\begin{array}{l}
\eta=\left(\frac{b}{v}\right)^{1 / 2}(1-c t)^{-1 / 2} y, \quad u=\frac{b x}{1-c t} f^{\prime}(\eta), \\
v=-\left(\frac{v b}{1-c t}\right)^{1 / 2} f(\eta) \\
T=T_{0}-T_{r e f} \frac{b_{1} x^{r}}{2 v}(1-c t)^{-m} \theta(\eta), \\
C=C_{0}-C_{r e f} \frac{b_{1} x^{r}}{2 v}(1-c t)^{-m} \phi(\eta),
\end{array}\right\},
$$

with the help of Eq. (9), Eqs.(4-6) are transformed into a system of ordinary non-linear coupled equations in the following form:

$$
\begin{aligned}
& f^{\prime \prime \prime}+f f^{\prime \prime}-f^{\prime 2}-S\left(\frac{\eta}{2} f^{\prime \prime}+f^{\prime}\right)-M f^{\prime}=0, \\
& \theta^{\prime \prime}+\operatorname{Pr}\left(f \theta^{\prime}-r f^{\prime} \theta\right)-\operatorname{Pr} S\left(\frac{\eta}{2} \theta^{\prime}+m \theta\right)+\operatorname{Pr} D f \phi^{\prime \prime}=0, \\
& \phi^{\prime \prime}+S c\left(f \phi^{\prime}-r f^{\prime} \phi\right)-S c S\left(\frac{\eta}{2} \phi^{\prime}+m \phi\right)+S r S c \theta^{\prime \prime}=0,
\end{aligned}
$$


where, the dashes denote to the partial derivatives with respect to the dimensionless variable $\eta$. Also, the transformed boundary conditions in Eqs. (7) and (8) can be written in the following form:

$$
\left.\begin{array}{l}
f(0)=0, \quad f^{\prime}(0)=1, \quad \theta(0)=1, \quad \phi(0)=1, \\
f\left(\eta_{\infty}\right)=\frac{S}{2} \eta_{\infty}, \quad f^{\prime \prime}\left(\eta_{\infty}\right)=\sigma_{1} \theta\left(\eta_{\infty}\right)+\sigma_{2} \phi\left(\eta_{\infty}\right), \\
\theta^{\prime}\left(\eta_{\infty}\right)=0, \quad \phi^{\prime}\left(\eta_{\infty}\right)=0 .
\end{array}\right\} .
$$

The dimensionless parameters are defined as follows:

$S=c / b$ the unsteadiness parameter, $M=\varepsilon B_{0}^{2}(1-c t) / \rho b$ the Magnetic parameter, $D f=D_{m} k_{T} C_{r e f} / c_{m} c_{p} v T_{r e f}$ the Dufour number, $S r=D_{m} k_{T} T_{r e f} / T_{m} v C_{\text {ref }}$ the Soret number, $S c=v / D_{m}$ the Schmidt number, $\operatorname{Pr}=\rho v c_{p} / k$ the Prandtl number, $\sigma_{1}=\sigma_{0} \gamma_{T} T_{r e f} b_{1} r x^{r-2}(1-c t)^{3 / 2-m} / 2 \mu \sqrt{b^{3} v}$ the temperature surface tension parameter, $\sigma_{2}=\sigma_{0} \gamma_{C} C_{r e f} b_{1} r x^{r-2}(1-c t)^{3 / 2-m} / 2 \mu \sqrt{b^{3} v}$ the concentration surface tension parameter.

The similarity distance at the free surface is $\eta_{\infty}$. This can be expressed as a function of the film thickness $h(t)$ in the following form

$$
h(t)=\eta_{\infty}\left(\frac{b}{v}\right)^{-1 / 2}(1-c t)^{1 / 2} .
$$

The evaluation of $\eta_{\infty}$ is considered as an integral part of the present boundary value problem. The local skin friction $C f_{x}$, the local Nusselt number $N u_{x}$ and the local Sherwood number $N m_{x}$ are the major physical quantities of the present problem. These quantities can be expressed by the functions $f, \theta$ and $\phi$ as follows:

$$
\begin{aligned}
& C f_{x}=-\left.\frac{2 \mu}{\rho U_{s}^{2}} \frac{\partial u}{\partial y}\right|_{y=0}=-2 \operatorname{Re}_{x}^{-1 / 2} f^{\prime \prime}(0), \\
& N u_{x}=-\left.\frac{x}{T_{s}-T_{0}} \frac{\partial T}{\partial y}\right|_{y=0}=-\operatorname{Re}_{x}^{1 / 2} \theta^{\prime}(0), \\
& N m_{x}=-\left.\frac{x}{C_{s}-C_{0}} \frac{\partial C}{\partial y}\right|_{y=0}=-\operatorname{Re}_{x}^{1 / 2} \phi^{\prime}(0),
\end{aligned}
$$

where, the dimensionless quantity $\operatorname{Re}_{x}=\frac{U_{s} x}{v}$ is the local Reynolds number.

\section{Analytical Solution for the Steady Flow:}

In this section, the analytical solution for the case of the steady flow at some special values of the parameters is studied. The system of Eqs.(10-12) can be reduced to a simple form by taking $S=0$ with $S r=D f=\sigma_{1}=\sigma_{2}=0$ as follows:

$$
\begin{aligned}
& f^{\prime \prime \prime}+f f^{\prime \prime}-f^{\prime 2}-M f^{\prime}=0, \\
& \theta^{\prime \prime}+\operatorname{Pr}\left(f \theta^{\prime}-r f^{\prime} \theta\right)=0, \\
& \phi^{\prime \prime}+S c\left(f \phi^{\prime}-r f^{\prime} \phi\right)=0,
\end{aligned}
$$

with the boundary conditions

$$
\left.\begin{array}{l}
f(0)=0, \quad f^{\prime}(0)=1, \quad \theta(0)=1, \quad \phi(0)=1, \\
f^{\prime \prime}\left(\eta_{\infty}\right)=0, \quad \theta^{\prime}\left(\eta_{\infty}\right)=0, \quad \phi^{\prime}\left(\eta_{\infty}\right)=0 .
\end{array}\right\},
$$


The system of Eqs. (18-20), according to the boundary conditions (21), has an exact solution which is presented by Magyari and Chamkha [7] in the following form

$$
\begin{aligned}
& f(\eta)=\frac{1}{a}\left(1-e^{-a \eta}\right), \\
& \theta(\eta)=1-\frac{\operatorname{Pr}}{a^{2}}\left(1-e^{-a \eta}\right), \\
& \phi(\eta)=1-\frac{S c}{a^{2}}\left(1-e^{-a \eta}\right) .
\end{aligned}
$$

This solution is available only at $r=1$. Where, the constant $a$ is defined as $a=\sqrt{1+M}$. The previous analytical solution is employed to assess the accuracy of the numerical solution of this study. This comparison will be illustrated in the next section.

\section{Numerical Solution: Quasilinearization method (QLM)}

The set of Eqs. (10-12) are non-linear coupled ordinary differential equations. The QLM method is used to transform these equations into a system of linear differential equations, and then the method of complementary function is applied to solve the resulting equations. The yielding equations are solved with the fourth order Runge-Kutta method. The QLM was presented in details in Ref. [30]. To benefit the reader, the major algorithm of this method can be presented in the following sequence.

Defining the new variables $x_{1}-x_{7}$ as follows

$$
\begin{array}{lll}
x_{1}=f, & x_{2}=f^{\prime}, & x_{3}=f^{\prime \prime}, \\
x_{4}=\theta, & x_{5}=\theta^{\prime}, & x_{6}=\phi,
\end{array} \quad x_{7}=\phi^{\prime},
$$

By using the above new variables, the system of higher order differential Eqs. (10-12) and the boundary conditions (13) can be transformed into another system of first order differential equations by performing Taylor's series expansion and the final resulting equations can be written in the matrix form as follows:

$X^{\prime}=A X+B$,

The elements of the $(7 \times 7)$ - matrices $A$ and $(7 \times 1) B$ are defined as follows

$$
\left.\begin{array}{l}
a_{i j}=\left(\frac{\partial g_{i}}{\partial x_{j}}\right)_{x^{k}} \\
b_{i}=g_{i}\left(x_{j}^{k}, \eta\right)-\sum_{j=1}^{7}\left(\frac{\partial g_{i}}{\partial x_{j}}\right)_{x^{k}} x_{j}^{k}
\end{array}\right\} \quad \text { for } \quad i, j=1,2, \ldots, 7 .
$$

The values of the elements $a_{i, j}$ and $b_{i}$ are defined in the Appendix. The system in Eq. (26) is linear and its general solution is obtained by using the method of complementary function, which determines the general solution as a particular and homogeneous solution. The boundary conditions for the particular solution and the homogeneous solutions are given as follows

(i) For the particular solution

$$
q_{i}^{(k+1)}(0)=(0,1,0,1,0,1,0),
$$

(ii) For the homogeneous solution

$$
\begin{aligned}
& U_{i}^{(1)}(0)=(0,0,1,0,0,0,0), \\
& U_{i}^{(2)}(0)=(0,0,0,0,1,0,0), \\
& U_{i}^{(3)}(0)=(0,0,0,0,0,0,1) .
\end{aligned}
$$


Then, the general solution of Eq. (26) is given by

$$
x_{i}^{(k+1)}(\eta)=q_{i}^{(k+1)}(\eta)+\sum_{l=1}^{3} d_{l} U_{i}^{(l)}(\eta)
$$

where, $d_{1}, d_{2}$ and $d_{3}$ are the missing initial conditions and are determined with the help of the boundary conditions at $\eta_{\infty}$. The values of these constants are defined in the Appendix. The particular and the homogeneous part of the equations are solved by employing the fourth order Runge-Kutta method. The coefficients $a_{i j}$ and $b_{i}$ in Eq.(26) depend on the nominal trajectories $x_{i}^{(0)}(\eta)$. Hence, depending on the initial guess of nominal trajectories $x_{i}^{(0)}(\eta)$, the solution of Eq.(26) yields the neighboring trajectories $x_{i}^{(1)}(\eta)$. These neighboring trajectories are treated as nominal trajectories and the next neighboring trajectories are obtained. This process is continued until the convergence is obtained. The whole range is divided initially into sub-interval of width $\Delta \eta=0.01$. It is important to mention here that, the initial guess of the nominal values are based on a guessed function which must be satisfy all the boundary conditions of the problem. The value of $\eta_{\infty}$ is improved during the calculations.

The analytical solution, which illustrated in the previous section, is compared graphically with the numerical solution of the general case (that was obtained by the QLM) in Fig.2 and in Table 2. These tabulated results show a higher accuracy for the method depending on the choice of $\Delta \eta$. Also, Fig.2 shows an excellent agreement between the curves for the velocity, temperature and concentration.

\section{Results and Discussion}

The system of Eqs.(10-12), with the boundary conditions (13), is solved numerically by using QLM. The numerical values of the velocity, temperature, concentration, wall shear stress and the rate of heat and mass transfer are computed for different effective parameters. In order to verify the accuracy of the present results, the QL solution of the film thickness $\eta_{\infty}$ and the wall shear stress $f^{\prime \prime}(0)$ are compared with the previous results of Refs. [11] and [12] in Table 1. A good agreement is found in the comparisons. Also, the analytical solution, that obtained in Eqs. (22-24), is compared with the QL solution in Table 2 and in Fig.2. It is observed that the error is very small for different values of $\Delta \eta$, and the two solutions are compatible.

Table 1. Comparison for values of $\eta_{\infty}$ and $f^{\prime \prime}(0)$ at $M=0, \operatorname{Pr}=1, r=2, m=2 / 3$, and $D f=S r=S c=\sigma_{1}=\sigma_{2}=0$.

\begin{tabular}{cccc}
\hline \multicolumn{4}{c}{$\eta_{\infty}$} \\
\hline$S$ & Andersson et al. [12] & Mahmoud [11] & Present results \\
\hline 0.8 & 2.15199 & 2.1519950 & 2.15310189 \\
1.2 & 1.12778 & 1.1277815 & 1.12778232 \\
\hline \multicolumn{5}{c}{$f^{\prime \prime}(0)$} \\
\hline 0.8 & -1.24581 & -1.245810 & -1.24547975 \\
1.2 & -1.27917 & -1.279170 & -1.27481814 \\
\hline
\end{tabular}


Table 2. Comparison for values of $f^{\prime \prime}(0)$ for the exact and numerical solutions at $r=1, m=2 / 3$, and $S=D f=S r=\sigma_{1}=\sigma_{2}=0$.

\begin{tabular}{ccccccc}
\hline \multicolumn{7}{c}{$f^{\prime \prime}(0)$} \\
\hline$\Delta \eta$ & $\operatorname{Pr}$ & $M$ & $S c$ & $\begin{array}{c}\text { Exact Solution } \\
\text { Eqs.(22-24) }\end{array}$ & $\begin{array}{c}\text { Numerical Solution } \\
\text { QLM }\end{array}$ & Error \\
\hline 0.03 & 0.5 & 5 & 0.5 & -2.44948974278 & -2.44948974278 & $8.30314 \times 10^{-12}$ \\
& 0.8 & 10 & 0.1 & -3.31662479036 & -3.31662479036 & $2.22045 \times 10^{-15}$ \\
\hline 0.02 & 0.5 & 20 & 0.5 & -4.58257569496 & -4.58257569496 & $4.44089 \times 10^{-15}$ \\
& 0.8 & 10 & 0.1 & -3.31662479036 & -3.31662479030 & $6.07834 \times 10^{-11}$ \\
\hline 0.01 & 0.6 & 6 & 0.3 & -2.64575131107 & -2.64550242449 & $2.48887 \times 10^{-4}$ \\
& 0.5 & 20 & 0.5 & -4.58257569496 & -4.58257554688 & $2.48887 \times 10^{-4}$ \\
\hline
\end{tabular}

Fig.3 illustrates that the unsteadiness parameter $S$ reduces the dimensionless film thickness $\eta_{\infty}$. Also, for critical value of the unsteadiness parameter $(S=2)$ the film thickness tends to zero. This implies that the film thickness vanishes at this critical value and then the motion becomes impossible. This physical result can be explained by the value of the film thickness, as given in Eq. (14). Increasing the value of $S$ corresponds to an increases of the parameter $c$, which gives an imaginary value for the root $(1-c t)^{1 / 2}$. So, the motion becomes impossible at certain critical value. The same physical situation was obtained for the non-Newtonian power law fluid by Chien [4] and for second grade fluid by Hayat et al. [19].

One of the main objectives of this study is to discuss the surface tension effect, according to thermosolutal Marangoni convection effect, on the motion. The physical values of $\gamma_{T}$ and $\gamma_{C}$ denote to the gradient of the surface tension with both the heat and mass transfer. So, the increase of the temperature surface tension parameter $\sigma_{1}$ and the concentration surface tension parameter $\sigma_{2}$ means a decrease in the surface tension (according to the negative values of the coefficients $\gamma_{T}$ and $\gamma_{C}$ ). Consequently, the reduction of the surface tension, at the free surface, reduces the free surface rigidity and consequently increases its ability to movement. Therefore, the velocity increases as well as the momentum film thickness increases as shown in Figs.3 and 4. Also, a similar theoretical result was obtained by Chien [4]. The author deduced that the thermo capillary parameter increases the film thickness. Also, the effect of the unsteadiness parameter on the fluid velocity is shown in Fig. 4. As illustrated before, the fluid film thickness vanishes at the critical value of the unsteadiness parameter $(S \rightarrow 2)$.

Also, the effect of the surface tension coefficients on the heat and mass transfer is illustrated in Figs.8 and 11, respectively. It is assumed that $T<T_{0}$ and $C<C_{0}$ throughout the boundary layer. Physically, increasing of both the temperature and the concentration gradients provokes a higher instability at the interface according to reducing the surface tension. This means that increasing both the temperature and the concentration surface tension coefficients $\sigma_{1}$ and $\sigma_{2}$ reduces the temperature and the concentration profiles, as shown in Figs.8 and 11. Similar result was obtained experimentally by Aubeterre et al.[31]. This experimental study deduced that the temperature gradient destabilizes the free surface. Higher temperature gradients destabilize the free surface and the movement becomes continuous. For the alcohol study, a sinuous wave was observed when temperature gradients are $15-17{ }^{\circ} c$ [26]. This means that the surface tension decreases and the substance evaporate easily when the temperature gradient occurs. This result was obtained previously by Chien [4] and Lin et. al. [8]. It was observed that the Marangoni convection reduced the free-surface 
temperature. Also, when the concentration at the interface is less than the concentration at the slot $\left(C<C_{0}\right)$ the surface tension at the free surface accordingly reduces. This implies that the surface tension is inversely proportional with the concentration.

Lorentz's force arises when a magnetic field is applied on an electrically conducting fluid. This resistive force retards the motion of the fluid and enhances its temperature. This effect is clear for the velocity in Fig.5 and for the temperature as well as the concentration in Figs. 8 and 10, respectively. Also, these figures illustrate that the magnetic field decreases the film thickness. This physical situation was found previously by Hayat et al. [19] for both the velocity and temperature profiles.

The effect of the Schmidt number $S c$ is highlighted in Figs.7 and 12 for the temperature and concentration distributions, respectively. The relative effect of momentum diffusion to particles diffusion is presented by Schmidt number. For $S c<1$, the particles diffusivity dominates. Decreasing the particles diffusivity produces an increase in the temperature gradients and means an increase in Schmidt number. So, it is observed that Schmidt number $S c$ enhances the temperature. Meanwhile, the higher values of Schmidt number correspond to a reduction in the particles diffusion. So, the concentration decreases with increasing $S c$.

Fig.6 illustrates that, the Prandtl number Pr reduces the temperature. An increase of Prandtl number means a slow rate of thermal-diffusion. This physically implies a decrease in the thermal boundary layer thickness. The same physical situation was previously cited in the literatures. Chien [4] observed that the free-surface temperature approaches unity in the high thermal diffusivity limit $(\operatorname{Pr} \rightarrow 0)$, whereas it diminishes in the high Pr (low thermal diffusivity) regime. Meanwhile, Prandtl number enhances the concentration profiles as shown in Fig.12.

Figs. 6,7,10 and 12 show the temperature and concentration distributions with collective variation in Soret $S r$ and Dufour $D f$ numbers. In all figures, the temperature and concentration profiles descend smoothly from a maximum value at the plate surface to the free stream. Soret number $S r$ represents the effect of temperature gradients on mass diffusion. Meanwhile, the effect of concentration gradients on thermal energy flux is described by Dufour number $D f$. It is observed from Fig. 6 that an increase in $D f$ from 0.1 to 0.9 (and $S r$ simultaneously decreases from 0.5 to 0.1 in Fig.7) enhances the temperature strongly. In Fig. 10, the concentration function $\phi$ increases as $D f$ decreases from 10 to 0.2 (and $S r$ simultaneously increases from 0.1 to 0.5 in Fig.12). So, we can conclude that the mass diffusion is enhanced evidently as a result of the temperature gradients. This physical result indicates that the diffusion is faster in both hotter and more rarefied gases, which well known in physics as Stokes-Einstein equation [32].

The unsteadiness parameter $S$ reduces both of the temperature and the concentration boundary layer thickness. Similar result was obtained by Mukhopadhyay [20]. This implies that the heat and mass transfer gradients decrease with increasing of the time according to the values of $S$ (and consequently with the power $m$ of the time) as shown in Figs.6, 9 and 10. Also, the surface temperature and the surface concentration always decrease moving away from the slot according to the horizontal distance $x$ (and consequently with the power $r$ of $x$ ). So, the temperature and the concentration profiles fall with rising $r$ as shown in Figs. 7 and 11.

Finally, Figs.13 and 14 illustrate the curves of the local skin friction $C f_{x}$, the local Nusselt number $N u_{x}$ and the local Sherwood number $N m_{x}$ versus the local Reynolds number $\operatorname{Re}_{x}$ at selected values of the unsteadiness parameter $S$. These figures confirm the same previous deduction that is the unsteadiness parameter has a critical value $(S=2)$. At this critical value the wall shear stress, the wall heat and mass fluxes 
curve approach to zero. Also, the unsteadiness parameter reduces the wall shear stress as well as the heat and mass fluxes.

\section{Conclusion}

In this paper, the effect of the thermosolutal Marangoni convection on the MHD unsteady flow is examined with taking into account the existence of the thermal diffusion and the diffusion thermo effects. The analytical solution of the steady state problem is obtained and compared with the numerical QLM solution. Also, the present results are compared with the previous results of Andersson et al. [12] and Mahmoud [11] for a special choice of some parameters. The effect of the Marangoni convection, in the present study, is compared with the previous experimental results of Aubeterre et al [31] in the discussion section. An agreement is found between the theoretical results of the present study and the previous experimental results which support the study. The main results can be concluded as follows:

1- The free surface loses its rigidity with reducing the surface tension and accordingly the flow movement increases. While, an increase in the temperature and the concentration provokes the free surface and makes it volatile easily, this coincides with the decreasing of the surface tension.

2- The unsteadiness parameter retards the film thickness growth and at a certain critical value of the unsteadiness parameter the motion, the heat and mass transfer are vanishing.

3- As well known, the transverse magnetic field retards the flow velocity and enhances both of the heat and mass transfer.

\section{Acknowledgment}

The author would like to bring profound thanks to the reviewers for their venerable efforts and valuable comments that have enriched the article.

\section{References:}

[1] L.G. Napolitano, Microgravity Fluid Dynamics : 2nd Levitch Conference, Washington, (1978).

[2] L.G. Napolitano, Marangoni boundary layers, in: Proc. 3rd European Symposium on Material Science in Space, Grenoble, ESA SP-142, June (1979).

[3] D.M. Christopher and B. Wang, Prandtl number effects for Marangoni convection over a flat surface, Int. J. Ther. Sci., 40, 564-570 (2001).

[4] Chien-Hsin Chen, Marangoni effects on forced convection of power-law liquids in a thin film over a stretching surface, Phy. Lett. A, 370, 51-57 (2007).

[5] I. Pop, A. Postelnicu, T. Grosan, Thermosolutal Marangoni forced convection boundary layers, Meccanica 36, 555-571 (2001).

[6] C. Jiao, L. Zheng, , L. Ma, MHD thermosolutal marangoni convection heat and mass transport of power law fluid driven by temperature and concentration gradient, AIP Adv. 5, 087160 (2015).

[7] E. Magyari and A.J. Chamkha, Exact analytical results for the thermosolutal MHD Marangoni boundary layers, Int. J. Ther. Sci., 47, 848-857 (2008).

[8] Y. Lin, L. Zheng, X. Zhang, Magnetohydrodynamics Thermocapillary Marangoni Convection Heat Transfer of Power-Law Fluids Driven by Temperature Gradient, J. Heat Trans., 135 (2013).

[9] R. Cortell, Toward an understanding of the motion and mass transfer with chemically reactive species for two classes of viscoelastic fluid over a porous stretching sheet, Chem. Engng. Process. 46, 982-989 (2007).

[10] N.S. Elgazery and M.A. Hassan, The effects of variable fluid properties and magnetic field on the flow of non-Newtonian fluid film on an unsteady stretching sheet through a porous medium, Commun. Numer. Meth. Engng. 24, 2113-2129 (2008).

[11] M.A.A. Mahmoud, Thermal radiation effects on the flow and heat transfer in a liquid film on an unsteady stretching sheet, Int. J. Num. Meth. Fluids, 67, 1692-1701 (2011) 
[12] H.I. Andersson, J.B. Aarseth, B.S. Dandapat, Heat transfer in a liquid film on an unsteady stretching surface, Int. J. Heat Mass Trans. 43, 69-74 (2000).

[13] B.S. Dandapat, B. Santra, H.I. Andersson, Thermocapillarity in a liquid film on an unsteady stretching surface, Int. J. Heat Mass Trans. 46, 16, 3009-3015 (2003).

[14] M.E. Ali and E. Magyari, Unsteady fluid and heat flow induced by a submerged stretching surface while its steady motion is slowed down gradually, Int. J. Heat Mass Trans. 50 (1-2) 188-195 (2007).

[15] B.S. Dandapat, B. Santra and K. Vajravelu, The effects of variable fluid properties and thermocapillarity on the flow of a thin film on an unsteady stretching sheet, Int. J. Heat Mass Trans. 50 (5-6) 991-996 (2007).

[16] E.M.A. Elbashbeshy and M.A.A. Bazid, Heat transfer over an unsteady stretching surface, Heat Mass Trans. 41, 1-4 (2004).

[17] S. Sharidan, T. Mahmood and I. Pop, Similarity solutions for the unsteady boundary layer flow and heat transfer due to a stretching sheet, Int. J. Appl. Mech. Eng. 11, 647-654 (2006).

[18] R. Tsai, K.H. Huang and J.S. Huang, Flow and heat transfer over an unsteady stretching surface with a non-uniform heat source, Int. Commun. Heat Mass Trans. 35, 1340-1343 (2008).

[19] T. Hayat, S. Saif and Z. Abbas, The influence of heat transfer in an MHD second grade fluid film over an unsteady stretching sheet, Phys. Lett. A 372, 5037-5045 (2008).

[20] S. Mukhopadhyay, Effect of thermal radiation on unsteady mixed convection flow and heat transfer over a porous stretching surface in porous medium, Int. J. Heat Mass

Trans. 52, 3261-3265 (2009).

[21] S. Mukhopadhyay, Heat transfer analysis of unsteady flow over a porous stretching surface embedded in a porous medium in presence of thermal radiation, Acta Tech. 56, 115-124 (2011).

[22] S. Mukhopadhyay, Effects of slip on unsteady mixed convective flow and heat transfer past a porous stretching surface, Nuc. Eng. Design 241, 2660-2665 (2011).

[23] A.J. Chamkha, A.M. Aly and M.A. Mansour, Similarity solution for unsteady heat and mass transfer from a stretching surface embedded in a porous medium with suction/injection and chemical reaction effects, Chem. Eng. Commun. 197, 846-858 (2010).

[24] K. Bhattacharyya, S. Mukhopadhyay and G.C. Layek, Slip effects on an unsteady boundary layer stagnation-point flow and heat transfer towards a stretching sheet, Chinese Phys. Lett. 28 (9) 094702, (2011).

[25] N. Sandeep, Effect of aligned magnetic field on liquid thin film flow of magnetic-nanofluids embedded with graphene nanoparticles, Adv. Powder Tech., 28, 865-875 (2017).

[26] G. Kumaran, N. Sandeep, M.E. Ali, Computational analysis of magnetohydrodynamic Casson and Maxwell flows over a stretching sheet with cross diffusion, Res. Phys., 7, 147-155 (2017).

[27] M.E. Ali , N. Sandeep, Cattaneo-Christov model for radiative heat transfer of magnetohydrodynamic Casson-ferrofluid: A numerical study, Res. Phys. 7, 21-30 (2017).

[28] M. Jayachandra Babu, N. Sandeep, UCM flow across a melting surface in the presence of double stratification and cross-diffusion effects, J. Mol. Liq., 232, 27-35 (2017).

[29] I-Chung Liu, Helge I. Andersson, Heat transfer in a liquid film on an unsteady stretching sheet, Int. J. Ther. Sci., 47, 766-772 (2008).

[30] S. M. Robert and J. S. Shipman., Two-point boundary value problem shooting methods, American Elsevier Publishing company, Inc. (1972).

[31] A. D'Aubeterre, R. Da Silva and M.E. Aguilera, Experimental study on Marangoni effect induced by heat and mass transfer, Int. Comm. Heat Mass Trans., 32, 677-684 (2005).

[32] Internet website: https://www.comsol.com/multiphysics/diffusion-coefficient. 


\section{$\underline{\text { Appendix }}$}

1-The elements of the matrices $A_{7 \times 7}$ and $B_{7 \times 1}$ are given as follows

$$
A=\left(\begin{array}{ccccccc}
0 & 1 & 0 & 0 & 0 & 0 & 0 \\
0 & 0 & 1 & 0 & 0 & 0 & 0 \\
a_{3,1} & a_{3,2} & a_{3,3} & 0 & 0 & 0 & 0 \\
0 & 0 & 0 & 0 & 1 & 0 & 0 \\
a_{5,1} & a_{5,2} & 0 & a_{5,4} & a_{5,5} & a_{5,6} & a_{5,7} \\
0 & 0 & 0 & 0 & 0 & 0 & 1 \\
a_{7,1} & a_{7,2} & 0 & a_{7,4} & a_{7,5} & a_{7,6} & a_{7,7}
\end{array}\right) \text { and } B=\left(\begin{array}{c}
0 \\
0 \\
b_{3} \\
0 \\
b_{5} \\
0 \\
b_{7}
\end{array}\right)
$$

where, the elements $a_{i, j}$ are defined as follows

$$
\begin{aligned}
& a_{3,1}=-x_{3}^{k}, \quad a_{3,2}=2 x_{2}^{k}+M+S, \\
& a_{3,3}=-x_{1}^{k}+\frac{S}{2} \eta, \\
& a_{5,2}= \frac{r}{1-\operatorname{Pr} S c S r D f}\left(\operatorname{Pr} x_{4}^{k}-S c D f \operatorname{Pr} x_{6}^{k}\right), \quad a_{5,4}=\frac{1}{1-\operatorname{Pr} S c S r D f}\left(\operatorname{Pr} r x_{2}^{k}+\operatorname{Pr} S m\right), \\
& a_{5,5}= \frac{1}{1-\operatorname{Pr} S c S r D f}\left(-\operatorname{Pr} x_{1}^{k}+\operatorname{Pr} \frac{S}{2} \eta\right) \\
& a_{5,6}= \frac{1}{1-\operatorname{Pr} S c S r D f}\left(-\operatorname{Pr} S c D f r x_{2}^{k}-\operatorname{Pr} S c D f S m\right), \\
& a_{5,7}= \frac{1}{1-\operatorname{Pr} S c S r D f}\left(\operatorname{Pr} S c D f x_{1}^{k}-\operatorname{Pr} S c D f S \frac{\eta}{2}\right), \\
& a_{7,1}= \frac{1}{1-\operatorname{Pr} S c S r D f}\left(S c S r \operatorname{Pr} x_{5}^{k}-S c x_{7}^{k}\right), \\
& a_{7,2}= \frac{r}{1-\operatorname{Pr} S c S r D f}\left(S c x_{6}^{k}-S c S r \operatorname{Pr} x_{4}^{k}\right) \\
& a_{7,4}= \frac{1}{1-\operatorname{Pr} S c S r D f}\left(-\operatorname{Pr} S c S r \quad r x_{2}^{k}-\operatorname{Pr} S c S r S m\right), \\
& a_{7,5}= \frac{1}{1-\operatorname{Pr} S c S r D f}\left(\operatorname{Pr} S c S r x_{1}^{k}-\operatorname{Pr} S c S r S \frac{\eta}{2}\right), \\
& a_{7,6}= \frac{1}{1-\operatorname{Pr} S c S r D f}\left(S c r x_{2}^{k}+S c S m\right), \\
& a_{7,7}=\frac{1}{1-\operatorname{Pr} S c S r D f}\left(-S c x_{1}^{k}+S c \frac{S}{2} \eta\right)
\end{aligned}
$$

and the elements $b_{i}$ are defined as follows

$b_{3}=x_{1}^{k} x_{3}^{k}-x_{2}^{k} x_{2}^{k}$,

$b_{5}=\frac{1}{1-\operatorname{Pr} S c S r D f}\left(\operatorname{Pr} x_{1}^{k} x_{5}^{k}-r \operatorname{Pr} x_{2}^{k} x_{4}^{k}+r \operatorname{Pr} S c D f x_{2}^{k} x_{6}^{k}-\operatorname{Pr} S c\right.$ Df $\left.x_{1}^{k} x_{7}^{k}\right)$ 
$b_{7}=\frac{1}{1-\operatorname{Pr} S c \operatorname{Sr} D f}\left(\operatorname{Sc} x_{1}^{k} x_{7}^{k}-r S c x_{2}^{k} x_{6}^{k}+r \operatorname{Pr} S c S r x_{2}^{k} x_{4}^{k}-\operatorname{Pr} S c \operatorname{Sr} x_{1}^{k} x_{5}^{k}\right)$.

2-The constants $d_{1}, d_{2}, d_{3}$ are given as follows

$$
d_{1}=\frac{\Delta_{1}}{\Delta}, \quad d_{2}=\frac{\Delta_{2}}{\Delta}, \quad d_{3}=\frac{\Delta_{3}}{\Delta},
$$

where

$$
\begin{aligned}
\Delta & =U_{3}^{(1)}\left(\eta_{\infty}\right)\left(U_{5}^{(2)}\left(\eta_{\infty}\right) U_{7}^{(3)}\left(\eta_{\infty}\right)-U_{5}^{(3)}\left(\eta_{\infty}\right) U_{7}^{(2)}\left(\eta_{\infty}\right)\right) \\
& -U_{3}^{(2)}\left(\eta_{\infty}\right)\left(U_{5}^{(1)}\left(\eta_{\infty}\right) U_{7}^{(3)}\left(\eta_{\infty}\right)-U_{5}^{(3)}\left(\eta_{\infty}\right) U_{7}^{(1)}\left(\eta_{\infty}\right)\right) \\
& +U_{3}^{(3)}\left(\eta_{\infty}\right)\left(U_{5}^{(1)}\left(\eta_{\infty}\right) U_{7}^{(2)}\left(\eta_{\infty}\right)-U_{5}^{(2)}\left(\eta_{\infty}\right) U_{7}^{(1)}\left(\eta_{\infty}\right)\right), \\
\Delta_{1} & =\left(\sigma_{1} x_{4}^{(k)}\left(\eta_{\infty}\right)+\sigma_{2} x_{6}^{(k)}\left(\eta_{\infty}\right)-q_{3}^{(k+1)}\left(\eta_{\infty}\right)\right)\left(U_{7}^{(3)}\left(\eta_{\infty}\right) U_{5}^{(2)}\left(\eta_{\infty}\right)-U_{7}^{(2)}\left(\eta_{\infty}\right) U_{5}^{(3)}\left(\eta_{\infty}\right)\right) \\
& -q_{5}^{(k+1)}\left(\eta_{\infty}\right)\left(-U_{3}^{(2)}\left(\eta_{\infty}\right) U_{7}^{(3)}\left(\eta_{\infty}\right)+U_{3}^{(3)}\left(\eta_{\infty}\right) U_{7}^{(2)}\left(\eta_{\infty}\right)\right) \\
& -q_{7}^{(k+1)}\left(\eta_{\infty}\right)\left(U_{5}^{(3)}\left(\eta_{\infty}\right) U_{3}^{(2)}\left(\eta_{\infty}\right)-U_{5}^{(2)}\left(\eta_{\infty}\right) U_{3}^{(3)}\left(\eta_{\infty}\right)\right), \\
\Delta_{2} & =\left(\sigma_{1} x_{4}^{(k)}\left(\eta_{\infty}\right)+\sigma_{2} x_{6}^{(k)}\left(\eta_{\infty}\right)-q_{3}^{(k+1)}\left(\eta_{\infty}\right)\right)\left(-U_{7}^{(3)}\left(\eta_{\infty}\right) U_{5}^{(1)}\left(\eta_{\infty}\right)+U_{7}^{(1)}\left(\eta_{\infty}\right) U_{5}^{(3)}\left(\eta_{\infty}\right)\right) \\
& -q_{5}^{(k+1)}\left(\eta_{\infty}\right)\left(U_{3}^{(1)}\left(\eta_{\infty}\right) U_{7}^{(3)}\left(\eta_{\infty}\right)-U_{3}^{(3)}\left(\eta_{\infty}\right) U_{7}^{(1)}\left(\eta_{\infty}\right)\right) \\
& -q_{7}^{(k+1)}\left(\eta_{\infty}\right)\left(-U_{5}^{(3)}\left(\eta_{\infty}\right) U_{3}^{(1)}\left(\eta_{\infty}\right)+U_{5}^{(1)}\left(\eta_{\infty}\right) U_{3}^{(3)}\left(\eta_{\infty}\right)\right), \\
\Delta_{3} & =\left(\sigma_{1} x_{4}^{(k)}\left(\eta_{\infty}\right)+\sigma_{2} x_{6}^{(k)}\left(\eta_{\infty}\right)-q_{3}^{(k+1)}\left(\eta_{\infty}\right)\right)\left(U_{7}^{(2)}\left(\eta_{\infty}\right) U_{5}^{(1)}\left(\eta_{\infty}\right)-U_{7}^{(1)}\left(\eta_{\infty}\right) U_{5}^{(2)}\left(\eta_{\infty}\right)\right) \\
& -q_{5}^{(k+1)}\left(\eta_{\infty}\right)\left(-U_{3}^{(1)}\left(\eta_{\infty}\right) U_{7}^{(2)}\left(\eta_{\infty}\right)+U_{3}^{(2)}\left(\eta_{\infty}\right) U_{7}^{(1)}\left(\eta_{\infty}\right)\right) \\
& -q_{7}^{(k+1)}\left(\eta_{\infty}\right)\left(U_{5}^{(2)}\left(\eta_{\infty}\right) U_{3}^{(1)}\left(\eta_{\infty}\right)-U_{5}^{(1)}\left(\eta_{\infty}\right) U_{3}^{(2)}\left(\eta_{\infty}\right)\right) .
\end{aligned}
$$

